\title{
Influencia de informalidad en titulación de tierras y planificación productiva, en cadenas agroexportadoras del Valle del Mantaro, Perú
}

\author{
Influence of informality in land titles and productive planning in \\ agro-export chains in the Mantaro Valley, Perú
}

\author{
William Rodríguez Giráldez' \\ Universidad Continental \\ wrodriguez@continental.edu.pe
}

\section{RESUMEN}

El objetivo fue determinar la influencia de la informalidad en la titulación de tierras sobre la planificación productiva en cadenas agroexportadoras del Valle del Mantaro. Investigación de alcance descriptivo-correlacional, no experimental, transeccional. Fueron encuestados 383 productores agrícolas de las provincias que comprenden el Valle del Mantaro: Huancayo, Concepción, Jauja y Chupaca. Se utilizaron estadísticos descriptivos como, distribución de frecuencias, tablas de contingencia, y ji al cuadrado para prueba de hipótesis. Entre los resultados se tiene que, 1) En informalidad en titulación de tierras, $61 \%$ de productores tienen título de propiedad y $2 \%$ en trámite; $95,7 \%$ saben de beneficios del título de propiedad; $55 \%$ manifiestan que terrenos son de su propiedad; $45 \%$ consideran que parte de sus terrenos los alquilan; 2) En planificación productiva, $52 \%$ de productores deciden qué sembrar en función a la demanda y mejor precio de mercado, $62 \%$ mejoraron resultados gracias a la planificación, $23 \%$ lograron metas de producción entre el $75 \%$ y $100 \%$, solo $19 \%$ mejoraron su productividad esperada y $47 \%$ mejoraron rangos inferiores a $25 \%$; además el $87 \%$ consideran que en la planificación de la producción deben participar productores y empresas. En conclusión, participar en cadenas productivas no depende de la tenencia de terrenos con título de propiedad. El trabajo asociativo no depende de la decisión de sembrar, de la planificación, de mejoras de productividad, ni de participación en procesos de planificación.

Palabras clave: Titulación de tierras, planificación productiva, trabajo asociativo, cadenas productivas.

\section{ABSTRACT}

The objective was to determine the informality influence of land ownership on the productive planning in the Mantaro Valley agro-export chains. The research has a descriptive-correlational, not experimental, transactional scope. 383 agricultural producers from the Mantaro Valley provinces: Huancayo, Concepcion, Jauja and Chupaca were surveyed. Descriptive statistics as frequency distribution, contingency tables and chi-squared distribution for hypothesis testing were used. The results are, 1) In the land ownership informality, $61 \%$ of producers have title deed and $2 \%$ are in process; 95,7 \% know about title deed benefits; $55 \%$ state which lands are their property; $45 \%$ consider they rent part of their lands; 2 ) In productive planning, $52 \%$ of producers decide what to seed based on the demand and the best market prices, $62 \%$ improve results because of planning, $23 \%$ achieve production targets between $75 \%$ and 100 $\%$, only $19 \%$ improve their expected productivity and $47 \%$ improve lower ranges to $25 \%$; also 87 $\%$ consider that in production planning they should involve agricultural producers and businesses. In conclusion, participation in productive chains doesn' $t$ depend on the lands possession with title deed. Partnership working isn't depend on the decision to seed, planning, productivity improvements, neither participation in planning processes.

Keywords: Land ownership, productive planning, partnership working, productive chains.

Historial del artículo:

Recibido: 29 de noviembre de 2014. Aprobado: 26 de mayo de 2015. Disponible en línea: 30 de junio de 2015

1 Magíster en Administración, Decano de la Facultad de Ciencias de la Empresa de la Universidad Continental. 


\section{INTRODUCCIÓN}

En la sierra peruana, se han formado por muchos años, cadenas productivas agropecuarias, promovidas por las ONG, instituciones de desarrollo, organismos públicos, etc., y se ofrecieron servicios de desarrollo como capacitación, asistencia técnica, articulación al mercado, financiamiento, etc.; sin embargo, al terminar el proyecto, no se lograron los resultados esperados y estas ya no operan o ya no existen, como señalan Avendaño et al. (1) en la cadena productiva de tuna y cochinilla en Ayacucho, que organizó a cerca de 1100 productores en 45 comités y a 20 comités de tuna-fruta, de los cuales permanecen solo el $5 \%$.

Benavides (2) plantea que siempre está latente el tema de la desintegración, estas asociaciones todavía son débiles institucionalmente. Antes, Prompex les pagaba al articulador y ahora que ya no cuentan con ese apoyo y todas las empresas deben aportar algo económicamente, empiezan a surgir las divisiones.

Scott (3) sostiene que una de las limitantes del desarrollo de clúster en el Perú ha sido el minifundismo y los altos costos de transacción que eso genera, para lograr la coordinación necesaria entre productores, vendedores de insumos, y procesadores. Otra limitante es la falta de financiamiento para pequeños productores y la práctica entre ciertos productores de no respetar contratos.

Las lecciones aprendidas de la experiencia con cadenas productivas de alcachofa, según Scott (3), fueron: 1) que el proceso de innovación comercial requiere del desarrollo de relaciones de coordinación y de confianza entre diferentes participantes en la cadena de valor y eso requiere tiempo; 2) la falta de títulos de propiedad de la tierra, entre otras cosas, que limita la capacidad de los agricultores de acceder al crédito para mejorar sus equipos, captar ciertas economías de escala en la producción de alcachofa y seguir mejorando su competitividad, y negociar contratos de uso y usufructo de la tierra con inversionistas exportadores; 3) más y mejor información y capacitación a las entidades financieras podrían ayudar en expandir el flujo de crédito hacia los diferentes miembros de la cadena de valor, pero eso requiere un esfuerzo institucional sostenido.

Dadas estas experiencias y estudio de las cadenas de valor agrícolas, Scott (4) identifica dos megaenfoques: el vertical y el horizontal o territorial. En el vertical, el análisis se concentra en la relación entre diferentes etapas, procesos y actores, desde la producción hasta el consumo final por un solo producto. Los temas de interés son: 1) la eficiencia técnica-económica: introduciendo nuevas tecnologías se pueden reducir costos y dirigir los márgenes al beneficio de los trabajadores, productores y consumidores; 2) infraestructura, tanto pública como privada; 3) capacitación en múltiples dimensiones: uso de tecnología disponible, negociación sobre los términos de compra venta, temas de gerencia de una organización; 4) la gobernanza o si la cadena de valor es una en que los productores son líderes en el proceso de cambio o son los actores más cercanos a los usuarios finales los que tienen mayor influencia sobre qué, cómo, dónde y cuándo hay que producir lo que se produce; 5$)$ políticas y procesos empresariales, o cómo cambiando el dónde y el quién compra los productos o la materia prima, se puede mejorar los ingresos de los agricultores y la calidad para los usuarios/clientes finales; 6) políticas gubernamentales, por ejemplo aranceles, impuestos, regulaciones; y 7) en múltiples formas, el medio ambiente, el uso de recursos naturales (renovables y no renovables), la protección de la biodiversidad, el cambio climático. Vale destacar que dichos temas no son mutuamente exclusivos.

El enfoque horizontal o territorial se refiere a cadenas de valor múltiples, sea por la misma materia prima (café) o grupos de cultivos en su conjunto (cultivos andinos), ubicadas en un territorio particular (región o distrito o corredor). El énfasis está basado más en la oferta-que sea actual (lo que se produce en la zona) - potencial (lo que se podría producir bajo el mismo clima, suelo, mano de obra)- que en la demanda, aunque en la práctica esto cambia rápidamente como parte del proceso dinámico asociado con el uso de este enfoque.

Scott (4) también plantea que "por las intervenciones verticales, implica reconocer que no a todos los pequeños productores les interesa modificar sus hábitos de siembra y cosecha para lograr una producción agrícola más intensiva y netamente comercial, ni que todos ellos producen los cultivos que le interesa a una u otra empresa privada". Sostiene que buena parte de la decisión sobre qué cadenas de valor enfocar, además de qué hacer al respecto, tiene que ver con quién es el promotor de dichas iniciativas. Puede ser una empresa, una organización no gubernamental (ONG) o un centro de investigación internacional. Típicamente, los promotores deciden los productos y lugares donde se van a trabajar y establecen los parámetros de los términos de interacción, pero ofreciendo a la vez elementos e incentivos a sus colaboradores (4).

Los productores del Valle del Mantaro tienen una cultura organizacional, definida por Schein (5) como "un modelo de presunciones básicas -inventadas, descubiertas o desarrolladas por un grupo dado al ir aprendiendo a enfrentarse con sus problemas de adaptación externa e integración internas- que hayan ejercido influencia como para ser consideradas válidas y, en consecuencia, ser enseñadas a los nuevos miembros como el modo correcto de percibir, pensar y sentir esos problemas". Esta concepción se pretende 
estudiar en el presente trabajo, para encontrar qué otros factores influyen en la formación, desarrollo y permanencia de cadenas productivas en el mercado, cuáles son según Van Muijen et al. (6) "los valores centrales, normas de conducta, artefactos y patrones de actividad que rigen la forma en que la gente en una organización interactúa con los demás e invierte energía en su trabajo y en la organización en general".

Peiró (7) clasifica la cultura de una organización formada por los siguientes contenidos: 1) nivel observable referido a los productos de la cultura, el lenguaje, los rituales, sanciones, normas, patrones de conducta, costumbres, símbolos, artefactos laborales, clima, mitos, historias, leyendas, espacios físicos, mobiliario, decoración, ropajes y vestidos, recompensas, etc.; 2) nivel apreciativo y valorativo, permite justificar, dar razón e interpretar los productos mencionados en el nivel anterior, e incluye valores, filosofía, ideologías, expectativas, actitudes, predisposiciones, sistema apreciativo, conocimiento, perspectivas, prioridades y significados; y 3) nivel fundante, integrado por supuestos y creencias básicas.

Gómez B., Mercedes (8) en su tesis doctoral Cultura en las Pymes del sector juguete y auxiliares cita a Alonso (9) quien categoriza los elementos culturales en dos grupos: a) el núcleo de la cultura, que corresponde a valores, creencias compartidas, entendimientos comunes, presupuestos, ideologías, filosofías; y b) las formas culturales, que son el aspecto expresivo de las acciones humanas: ritos, rituales, historias, leyendas, lenguaje especializado, patrones de comportamiento $y$, de forma general, se puede aplicar al resultado de las acciones humanas que además de cumplir con determinados fines técnicos y prácticos expresan un conjunto de significados culturales.

Para Schneider, Brief y Guzzo (10), la cultura organizacional puede ser cambiada a través de un cambio en el clima, definido como los tangibles que produce una cultura, los tipos de cosas que suceden alrededor de los empleados y que estos son capaces de describir, mientras que consideran la cultura como las creencias y valores firmemente implantados en una organización. El cambio solamente puede darse y mantenerse cambiando las políticas, prácticas, procedimientos y rutinas diarias, que alterarán las creencias y valores que guían las acciones de los empleados. Comunicar nuevos valores y creencias requiere cambiar tangibles, es decir, las miles de cosas que definen el clima, que definen el día a día de una organización.

Por ello se formulan interrogantes como: ¿̇por qué las cadenas productivas no permanecen en el mercado, si recibieron servicios de desarrollo como capacitación, asistencia técnica y promoción de mercado?, ¿qué otras razones diferentes a las técnicas, mercadeo y financieras, influyen en su sostenimiento y desarrollo?, ¿̇cómo influyen dos factores culturales, relacionados a la informalidad en la titulación de tierras y a la planificación productiva, en el trabajo asociativo de cadenas productivas en el Valle del Mantaro, Junín, Perú?. Los resultados de este estudio servirán para el manejo adecuado de estos factores culturales o cultura organizacional en la formación, permanencia en el mercado, crecimiento y desarrollo de cadenas productivas de agroexportación, a través de un proceso de cambio de la cultura organizacional y lograr, como consecuencia, mejorar la calidad de vida de los productores.

\section{MATERIAL Y MÉTODOS}

La investigación es descriptiva, no experimental, transeccional o transversal de alcance correlacional (11).

El universo de estudio fue la población rural del Valle del Mantaro, de 132459 habitantes, según el INEI (12), y que comprende las provincias de Huancayo (41\%), Concepción (22\%), Jauja (21\%) y Chupaca (16\%). La muestra fue de 383 productores agrícolas del referido valle, calculado con un margen de error permitido de $5 \%$, un factor $P$ de 0,5 y $Q$ de 0,5 . Se aplicó un muestreo probabilístico aleatorio estratificado por el número de habitantes del sector rural de cada provincia.

La técnica de recolección de datos fue la encuesta y el instrumento usado, el cuestionario (11), con preguntas de tipo nominal, cerradas, ya que se trató de encontrar la percepción o creencia de los productores de cada factor cultural en relación con el trabajo asociativo en cadenas productivas. El procesamiento de los datos se realizó mediante software especializado, estadísticas como distribución de frecuencias y tablas de contingencias. Para probar las hipótesis, fueron aplicadas pruebas de ji al cuadrado orientadas a determinar la relación entre las variables, porque se trabajaron con escalas de medición nominal.

\section{RESULTADOS}

El trabajo de investigación formuló dos hipótesis: 1) La informalidad en la titulación de tierras influye negativamente en el trabajo asociativo de cadena productiva en el Valle del Mantaro, Junín; y 2) La planificación productiva influye positivamente en el trabajo asociativo de cadena productiva en el mencionado valle. Los resultados fueron obtenidos en función del análisis de cada una de estas dos hipótesis planteadas. 
Tabla $\mathrm{N}^{\circ}$ 1: Terrenos con título de propiedad y trabajo asociativo.

\begin{tabular}{lrrrrr}
\hline & \multicolumn{4}{c}{$\begin{array}{c}\text { Terrenos con } \\
\text { título de propiedad }\end{array}$} & \multirow{2}{*}{ Total } \\
\cline { 2 - 5 } & & Si & No & En trámite & \\
\hline \multirow{2}{*}{ Trabajo sociativo } & Si & $54 \%$ & $35 \%$ & $2 \%$ & $91 \%$ \\
\cline { 2 - 5 } Total & No & $6 \%$ & $2 \%$ & $0 \%$ & $9 \%$ \\
\hline
\end{tabular}

\section{Informalidad en la titulación de tierras}

Para probar la hipótesis 1, se determinó si el trabajo asociativo en cadena agroexportadora es independiente de las dimensiones consideradas en la variable informalidad: terrenos con título de propiedad, conocimiento de los beneficios de tener un título de propiedad, y propiedad de terrenos para la cadena agroexportadora.

En el primer caso se determinó si el trabajo asociativo depende del título de propiedad de los terrenos.

La tabla $\mathrm{N}^{\circ} 1$ muestra que el $61 \%$ de productores tienen terrenos con título de propiedad, $37 \%$ que no

Tabla № 2: Conoce beneficios de título de propiedad y trabajo asociativo.

\begin{tabular}{|c|c|c|c|c|}
\hline & & \multicolumn{2}{|c|}{$\begin{array}{l}\text { Conoce beneficios de } \\
\text { tener título de propiedad }\end{array}$} & \multirow{2}{*}{ Total } \\
\hline & & $\mathrm{Si}$ & No & \\
\hline \multirow{2}{*}{ Trabajo sociativo } & $\mathrm{Si}$ & $88 \%$ & $3 \%$ & $91 \%$ \\
\hline & No & $8 \%$ & $1 \%$ & $9 \%$ \\
\hline Total & & $96 \%$ & $4 \%$ & $100 \%$ \\
\hline
\end{tabular}

tienen y $2 \%$ que mantienen en trámite. Utilizando el nivel de significación de 0,05 y 2 grados de libertad se obtiene un X2 de prueba de 2,284, inferior a ii al cuadrado crítico o de tabla de 5,991. Por tanto, se concluye que el trabajo asociativo de cadenas agroexportadoras no depende de la titulación de los terrenos.

Segundo, se determinó si el trabajo asociativo depende del conocimiento de los beneficios de tener título de propiedad.

La tabla $\mathrm{N}^{\circ} 2$ indica que el $96 \%$ de productores saben de los beneficios de tener título de propiedad y que solo $4 \%$ no saben. Utilizando el nivel de significación de 0,05 y 1 grado de libertad se obtiene un X2 de prueba de 5,718 superior a ji al cuadrado crítico o de tabla de 3,841, por lo que se concluye que el trabajo asociativo de las cadenas agroexportadoras depende del conocimiento de los beneficios del título de propiedad de los terrenos.

Tercero, se determinó si el trabajo asociativo en cadena agroexportadora depende de la situación de los terrenos agrícolas para formar una cadena de este tipo.

La tabla $\mathrm{N}^{\circ} 3$ muestra que el $55 \%$ de productores indican que todos los terrenos donde siembran son de su propiedad; que el $11 \%$ de ellos expresan que una cuarta parte les pertenece y el resto los alquila; que el $22 \%$ señalan que la mitad son suyos y el resto también los alquila; y que el $12 \%$ admiten que todos los terrenos son alquilados. Utilizando el nivel de significación de 0,05 y 3 grados de libertad se obtiene un X2 de prueba 0,336 , inferior a ji al cuadrado crítico o de tabla de 7,815. El resultado en este caso es que el trabajo asociativo no depende de la situación de los terrenos para cadena agroexportadora.

Las pruebas de ii al cuadrado establecen que la participación en una cadena productiva no depende del hecho de tener título de propiedad, ni de la situación de los terrenos, ya que para participar en esta cadena no necesariamente se requiere que todos los terrenos cuenten con título de propiedad, seguramente para solicitar créditos bancarios. Esto se puede explicar porque en los últimos años las empresas articuladoras de cadenas productivas, financiaron $100 \%$ de costos de producción y no se ha requerido de créditos para los productores, pero esta solución elevó el nivel de riesgo para la empresa articuladora, y como consecuencia realizaron sistemas de control de producción más exigentes que no fue del agrado de los productores. Además, los riesgos se vieron incrementados por la débil participación y poco involucramiento de los productores. Por lo tanto se acepta la hipótesis de que la informalidad en la

Tabla № 3: Situación de terrenos agrícolas para formar cadena agroexportadora y trabajo asociativo.

\begin{tabular}{lcccccc}
\hline & \multicolumn{5}{c}{ Situación de terrenos agrícolas } \\
\cline { 2 - 7 } & & $\begin{array}{c}\text { Todos son de } \\
\text { mi propiedad }\end{array}$ & $\begin{array}{c}\text { Una cuarta parte son } \\
\text { míos el resto alquilo }\end{array}$ & $\begin{array}{c}\text { La mitad son míos } \\
\text { el resto alquilo }\end{array}$ & $\begin{array}{c}\text { Alquilo todos } \\
\text { los terrenos }\end{array}$ & Total \\
\hline \multirow{2}{*}{ Trabajo sociativo } & $\mathrm{Si}$ & $50 \%$ & $10 \%$ & $20 \%$ & $11 \%$ & $91 \%$ \\
\cline { 2 - 8 } Total & $5 \%$ & $1 \%$ & $2 \%$ & $1 \%$ & $9 \%$ \\
\hline
\end{tabular}


Tabla № 4: Decisión de sembrar y trabajo asociativo.

\begin{tabular}{|c|c|c|c|c|c|c|c|}
\hline & & \multicolumn{5}{|c|}{ Situación de terrenos agrícolas } & \multirow[b]{2}{*}{ Total } \\
\hline & & $\begin{array}{c}\text { Según que } \\
\text { producto se está } \\
\text { vendiendo más }\end{array}$ & $\begin{array}{l}\text { Según el } \\
\text { precio en el } \\
\text { mercado }\end{array}$ & $\begin{array}{c}\text { Por } \\
\text { recomendación de } \\
\text { familiar o amistad }\end{array}$ & $\begin{array}{l}\text { Siembro lo } \\
\text { mismo conozco } \\
\text { el cultivo }\end{array}$ & $\begin{array}{l}\text { No me puedo } \\
\text { arriesgar con } \\
\text { cultivos nuevos }\end{array}$ & \\
\hline \multirow{2}{*}{ Trabajo sociativo } & $\mathrm{Si}$ & $24 \%$ & $25 \%$ & $20 \%$ & $11 \%$ & $11 \%$ & $91 \%$ \\
\hline & No & $2 \%$ & $2 \%$ & $2 \%$ & $1 \%$ & $1 \%$ & $9 \%$ \\
\hline Total & & $25 \%$ & $27 \%$ & $22 \%$ & $12 \%$ & $12 \%$ & $100 \%$ \\
\hline
\end{tabular}

Tabla № 5: Resultados de la planificación productiva y trabajo asociativo.

\begin{tabular}{|c|c|c|c|c|c|c|c|}
\hline & & \multicolumn{5}{|c|}{ Resultados basados en planificación productiva } & \multirow[b]{2}{*}{ Total } \\
\hline & & $\begin{array}{c}\text { Totalmente de } \\
\text { acuerdo }\end{array}$ & $\begin{array}{c}\mathrm{De} \\
\text { acuerdo }\end{array}$ & $\begin{array}{c}\text { Más o menos } \\
\text { de acuerdo }\end{array}$ & $\begin{array}{c}\text { En } \\
\text { desacuerdo }\end{array}$ & $\begin{array}{c}\text { Totalmente en } \\
\text { desacuerdo }\end{array}$ & \\
\hline \multirow{2}{*}{ Trabajo sociativo } & $\mathrm{Si}$ & $5 \%$ & $55 \%$ & $30 \%$ & $3 \%$ & $0 \%$ & $94 \%$ \\
\hline & No & $0 \%$ & $2 \%$ & $4 \%$ & $0 \%$ & $0 \%$ & $6 \%$ \\
\hline Total & & $5 \%$ & $57 \%$ & $34 \%$ & $3 \%$ & $0 \%$ & $100 \%$ \\
\hline
\end{tabular}

Tabla № 6: Logro de metas de producción y trabajo asociativo.

\begin{tabular}{|c|c|c|c|c|c|c|c|}
\hline & & \multicolumn{5}{|c|}{ Se lograron metas de producción } & \multirow[b]{2}{*}{ Total } \\
\hline & & $100 \%$ & $\begin{array}{c}\text { Solo el } \\
75 \%\end{array}$ & $\begin{array}{c}\text { Solo el } \\
50 \%\end{array}$ & $\begin{array}{c}\text { Solo el } \\
25 \%\end{array}$ & $\begin{array}{c}\text { No se } \\
\text { lograron }\end{array}$ & \\
\hline \multirow{2}{*}{ Trabajo sociativo } & $\mathrm{Si}$ & $3 \%$ & $20 \%$ & $33 \%$ & $31 \%$ & $7 \%$ & $95 \%$ \\
\hline & No & $0 \%$ & $0 \%$ & $1 \%$ & $2 \%$ & $2 \%$ & $5 \%$ \\
\hline Total & & $3 \%$ & $20 \%$ & $34 \%$ & $33 \%$ & $9 \%$ & $100 \%$ \\
\hline
\end{tabular}

Tabla N $\mathrm{N}^{\circ}$ : Mejora de la productividad y trabajo asociativo.

\begin{tabular}{|c|c|c|c|c|c|c|c|c|}
\hline & & \multicolumn{6}{|c|}{ Mejoro la productividad } & \multirow[b]{2}{*}{ Total } \\
\hline & & Empeoró & $\begin{array}{c}\text { Igual que } \\
\text { antes }\end{array}$ & $\begin{array}{c}\text { Mejoró entre } \\
10 \text { a } 25 \%\end{array}$ & $\begin{array}{c}\text { Mejoró entre } \\
30 \text { a } 50 \%\end{array}$ & $\begin{array}{c}\text { Mejoró entre } \\
60 \text { a } 75 \%\end{array}$ & $\begin{array}{c}\text { Mejoró entre } \\
80 \text { a } 100 \%\end{array}$ & \\
\hline \multirow{2}{*}{ Trabajo sociativo } & $\mathrm{Si}$ & $1 \%$ & $11 \%$ & $31 \%$ & $34 \%$ & $16 \%$ & $3 \%$ & $95 \%$ \\
\hline & No & $0 \%$ & $2 \%$ & $2 \%$ & $2 \%$ & $0 \%$ & $0 \%$ & $5 \%$ \\
\hline Total & & $1 \%$ & $13 \%$ & $33 \%$ & $35 \%$ & $16 \%$ & $3 \%$ & $100 \%$ \\
\hline
\end{tabular}

Tabla № 8: Participación en la planificación de la producción y trabajo asociativo.

\begin{tabular}{|c|c|c|c|c|c|c|c|}
\hline & & \multicolumn{5}{|c|}{ Participación en la planificación de la producción } & \multirow[b]{2}{*}{ Total } \\
\hline & & $\begin{array}{c}\text { Debe planificar } \\
\text { empresa } \\
\text { exportadora }\end{array}$ & $\begin{array}{c}\text { Solo los que } \\
\text { aportamos } \\
\text { mas }\end{array}$ & $\begin{array}{l}\text { La mitad de } \\
\text { productores }\end{array}$ & $\begin{array}{l}\text { La mayoría } \\
\text { de } \\
\text { productores }\end{array}$ & $\begin{array}{c}\text { Todos los } \\
\text { productores y } \\
\text { empresa }\end{array}$ & \\
\hline \multirow{2}{*}{ Trabajo sociativo } & $\mathrm{Si}$ & $4 \%$ & $3 \%$ & $5 \%$ & $23 \%$ & $56 \%$ & $91 \%$ \\
\hline & No & $0 \%$ & $1 \%$ & $1 \%$ & $3 \%$ & $4 \%$ & $9 \%$ \\
\hline Total & & $5 \%$ & $4 \%$ & $6 \%$ & $27 \%$ & $60 \%$ & $100 \%$ \\
\hline
\end{tabular}

titulación de tierras de los productores agropecuarios incide negativamente en el trabajo asociativo de las cadenas productivas de agroexportación en el Valle del Mantaro, Junín.

\section{Planificación asociativa}

Para probar la hipótesis 2, que la planificación 
productiva influye positivamente en el trabajo asociativo en cadena agroexportadora en el Valle del Mantaro, Junín; se determinó si el trabajo asociativo en cadena agroexportadora es dependiente de las variables consideradas en la variable planificación productiva: decisión de sembrar, resultados de planificación productiva, logro de metas, mejora de la productividad, y participación en la planificación productiva.

Sobre si el trabajo asociativo depende de la decisión de sembrar, la tabla $\mathrm{N}^{\circ} 4$ indica que el $26 \%$ de productores deciden sembrar según qué producto se está vendiendo más en el mercado, el 27 \% según el precio en el mercado, el $15 \%$ por recomendación de un familiar o amistad, en tanto que el $29 \%$ siempre siembran lo mismo porque conocen el producto y el $3 \%$ no se pueden arriesgar con cultivos nuevos, lo que en suma significa que un $32 \%$ de productores son renuentes a cultivar nuevos productos que probablemente no arriesgarían su participación en una cadena agroexportadora. Los productores de Huancayo (33\%) y Concepción (18\%) son los más innovadores en la decisión de sembrar, y pueden adoptar con mayor facilidad nuevos cultivos por demandas de mercados extranjeros para formar cadenas agroexportadoras. Utilizando el nivel de significación de 0,05 y 4 grados de libertad se obtiene un X2 prueba 2,696, inferior a ji al cuadrado crítico o de tabla de 9,488. Por lo tanto, se concluye que el trabajo asociativo no depende de la decisión de sembrar o que no hay relación entre estas variables.

Respecto a si el trabajo asociativo depende de los resultados de la planificación productiva, la Tabla $\mathrm{N}^{\circ}$ 5 indica que el $62 \%$ están de acuerdo y totalmente de acuerdo, mientras que el $37 \%$ muestran sus dudas o están en desacuerdo. Utilizando el nivel de significación de 0,05 y 4 grados de libertad se obtiene un X2 prueba 6,545, inferior a ji al cuadrado crítico o de tabla de 9,488 . Queda establecido que trabajo asociativo no depende de los resultados de la planificación o que no hay relación entre estas variables.

En cuanto a si el trabajo asociativo depende del logro de metas de producción, la Tabla № 6 muestra que solo el $3 \%$ de productores creen que se logró el 100 $\%$ de las metas propuestas y que el $20 \%$ consideran que se alcanzó el $75 \%$; en tanto, hay un $67 \%$ que señalan que solo se logró entre $25 \%$ y $50 \%$, y un $10 \%$ que manifiestan que no se lograron las metas. Utilizando el nivel de significación de 0,05 y 4 grados de libertad se obtiene un X2 prueba 14,234, superior a ji al cuadrado crítico o de tabla de 9,488, por lo que se concluye que el trabajo asociativo sí depende de los resultados de la planificación o que sí hay relación entre estas variables.

En torno a si el trabajo asociativo depende de la mejora de la productividad, la tabla $N^{\circ} 7$ muestra que solo el 3 $\%$ de productores indican que la productividad mejoró entre el $80 \%$ y $100 \%$ con el trabajo asociativo, el 16 $\%$ que mejoró entre $60 \%$ y $75 \%$, el $35 \%$ que mejoró entre el $30 \%$ y $50 \%$ y el $47 \%$ que mejoró por debajo del $25 \%$. Utilizando el nivel de significación de 0,05 y 5 grados de libertad se obtiene un X2 prueba 7,688, inferior a ji al cuadrado crítico o de tabla de 11,070. Por lo tanto, se concluye que el trabajo asociativo no depende de los resultados de la planificación o que no hay relación entre estas variables.

Referente a si el trabajo asociativo depende de la participación en la planificación de la producción, la Tabla $N^{\circ} 8$ indica que el $60 \%$ de productores creen que todos (productores y empresa) deben participar en la planificación de la producción; que el $26 \%$ consideran que la mayoría de productores deben hacerlo, y que apenas el $4 \%$ aceptan que deben planificar solo la empresa agroexportadora. Utilizando el nivel de significación de 0,05 y 4 grados de libertad se obtiene un $\mathrm{X} 2$ prueba 7,327 , inferior a ji al cuadrado crítico o de tabla de 9,488; caso que permite concluir que el trabajo asociativo no depende de los resultados de la planificación o que no hay relación entre estas variables.

Con estas cinco pruebas, se determinó que el trabajo asociativo en cadena agroexportadora es dependiente solo del logro de metas a través de la planificación de producción, mas no de los otros componentes. Por lo tanto, se deduce que la planificación productiva no influye positivamente en el trabajo asociativo de cadena agroexportadora en el Valle del Mantaro, Junín.

La Figura $\mathrm{N}^{\circ} 1$ muestra los resultados de la prueba ji al cuadrado, prueba de tipo no paramétrica y fue usada porque en el proyecto se trabajó con escalas de medición nominal. Las variables en cuadrícula completa expresan que hay relación entre las variables con la variable trabajo asociativo en cadenas agroexportadoras, y las cuadrículas punteadas indican que no hay relación.

\section{DISCUSIÓN}

En la dimensión informalidad en la titulación de tierras, el $61 \%$ de productores manifiestan que tienen título de propiedad, $37 \%$ expresan que no y $2 \%$ que está en trámite. El 95,7\% de los productores saben de los beneficios de tener un título de propiedad y el $55 \%$ señalan que los terrenos que pondrían a una cadena agroexportadora son de su propiedad. Las pruebas de ii al cuadrado establecen que la participación en una cadena productiva en el Valle del Mantaro no depende del hecho de tener título de propiedad, ya que para participar en una cadena productiva no 
necesariamente se requiere que todos los terrenos sean de propiedad de los productores y tengan título de propiedad, probablemente para solicitar créditos bancarios, contrastando con lo que plantea Scott (3), la falta de títulos de propiedad de la tierra, entre otras cosas, limita la capacidad de agricultores de 1) acceder a crédito para mejorar sus equipos, captar ciertas economías de escala en la producción de alcachofa, y seguir mejorando su competitividad; y 2) negociar contratos de uso y usufructo de la tierra con inversionistas exportadores. Los resultados se pueden explicar porque en los últimos años las empresas articuladoras de cadenas productivas, que llegaron al valle, ofrecieron financiar hasta el $100 \%$ de los costos de producción. Sin embargo, esta solución elevó el nivel de riesgo para la empresa articuladora, y realizaron sistemas de control de producción más exigentes que no fueron del agrado de los productores, ellos siembran productos que se están vendiendo en el mercado local y $32 \%$ no desean cambiar a nuevos cultivos y la empresa articuladora trae la propuesta de productos nuevos para mercados de exportación, iniciativa que genera contradicciones en las decisiones de qué sembrar e involucrarse con el cultivo, que no despierta confianza en las nuevas propuestas de cultivo y por lo tanto el productor no se involucra con la nueva propuesta, considerando además lo que sostiene Laguna (13), que conocer los encadenamientos existentes, sus miembros, sus interrelaciones y sus fuentes de ventaja competitiva es un elemento importante para comprender el desarrollo de las economías locales. Sin embargo, un problema común en los estudios de clúster es que estos se definen a priori, sin un análisis cuantitativo o cualitativo suficiente, lo que impide identificar algunas relaciones clave para la región.

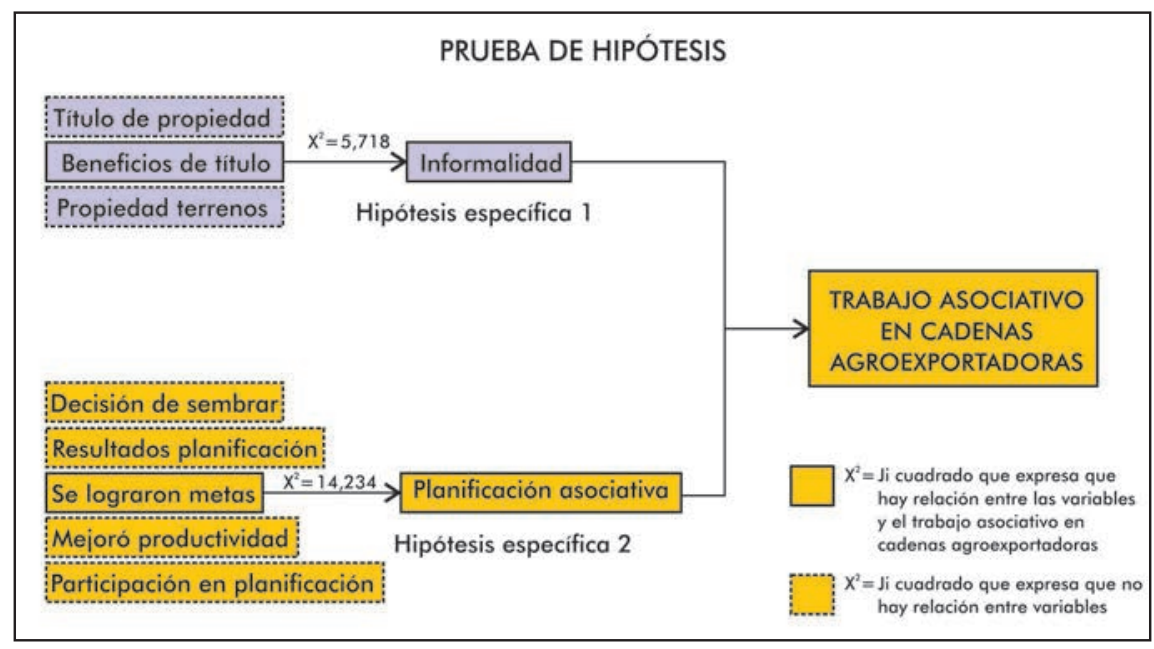

Figura $\mathrm{N}^{\circ}$ 1: Resultados de las pruebas de hipótesis utilizando Ji cuadrado.

además promovieron la débil participación y poco involucramiento de los productores.

En la dimensión planificación productiva, el estudio muestra que el $52 \%$ de los productores toman la decisión de sembrar según qué producto se está vendiendo más y por el mejor precio del mercado, e indican que están al tanto de lo que sucede en el mercado y $32 \%$ no desea cambiar el cultivo. La prueba de ji al cuadrado concluye que el trabajo asociativo en el Valle del Mantaro no depende de la decisión de sembrar o que no hay relación entre estas variables, que corrobora lo planteado por Scott (4) al respecto, que "por las intervenciones verticales, implica reconocer que no a todos los pequeños productores les interesa modificar sus hábitos de siembra y cosecha para lograr una producción agrícola más intensiva y netamente comercial, ni que todos ellos producen los cultivos que le interesa a una u otra empresa privada". En la cultura del productor del Valle del Mantaro se presenta una contradicción porque $52 \%$ de
El $62 \%$ de productores consideran que las mejoras fueron resultados de la planificación, sin embargo la prueba de ji al cuadrado define que el trabajo asociativo no depende de los resultados de la planificación o que no hay relación entre estas variables, porque los productores no estuvieron involucrados con los resultados programados o no creían en ellos.

En cuanto a si el trabajo asociativo depende de la mejora de la productividad, la Tabla $N^{\circ} 7$ muestra que solo el $3 \%$ de productores indican que la productividad mejoró entre el $80 \%$ y $100 \%$ con el trabajo asociativo, es decir se llegó a la meta; el $16 \%$ manifiesta que mejoró entre $60 \%$ y $75 \%$; el $35 \%$ que lo hizo entre el $30 \%$ y $50 \%$; y $47 \%$, que solo obtuvo mejoras inferiores al $25 \%$ de lo programado o no lo consiguió. Al no confiar en los proceso de planificación, los productores no se involucran en estos procesos, corroborando lo que plantea Scott (4), que "típicamente, los promotores deciden los productos y lugares donde se van a 
trabajar y establecen los parámetros de los términos de interacción, pero ofreciendo a la vez elementos e incentivos a sus colaboradores".

El $87 \%$ de productores consideran que deben participar todos los productores y la empresa en la planificación de la producción. Esto puede explicar lo que plantea Scott (4), que buena parte de la decisión sobre qué cadenas de valor enfocar además de qué hacer al respecto, tiene que ver con quién es el promotor de dichas iniciativas. Puede ser una empresa, una organización no gubernamental (ONG) o un centro de investigación internacional, y típicamente los promotores deciden los productos y lugares donde se van a trabajar y establecen los parámetros de los términos de interacción, pero ofreciendo a la vez elementos e incentivos a sus colaboradores (4), por ello la empresa que quiera promover una cadena productiva en el Valle del Mantaro, tiene que trabajar en la planificación productiva, considerando la participación conjunta con los productores, si desea involucramiento de ellos en el negocio.

Las pruebas ii al cuadrado concluyen que el trabajo asociativo de las cadenas agroexportadoras en el Valle del Mantaro no depende de la decisión de sembrar, de los resultados como consecuencia de la planificación, de la mejora de la productividad y de la participación en la planificación. Al no haber logrado los resultados planificados, los productores no tienen confianza en estos procesos, por lo que se deduce que la planificación asociativa no influye positivamente en el trabajo asociativo.

\section{REFERENCIAS BIBLIOGRÁFICAS}

1. Avendaño $E$, Roeland $R$, Risco $M$, Martínez $A$, et al. Conociendo la cadena productiva de tuna y cochinilla en Ayacucho. Ayacucho: Solid Perú; 2008: Disponible en: http://www. solidinternational.ch/wp-content/themes/solid/ sources/img/Conociendo-la-cadena-productivade-la-tuna-y-cochinilla-en-Ayacuchol.pdf

2. Benavides M. Asociatividad y Clusters. En: Documento de trabajo: Estudio sobre Cluster y Asociatividad. Lima: MTPE/PROMPYME. Disponible en: http://www.uss.edu.pe/uss/ eventos/JovEmp/pdf/ClusterdeCalzado.pdf

3. Gregory JS. Competitividad Agrícola y el desarrollo de cadenas y clústeres de valor en el Perú. Lima; 2011. Disponible en: http://idbdocs.iadb.org/ wsdocs/getdocument.aspx? docnum $=35839504$

4. Gregory JS. Agregando valor a las cadenas de valor. RAE. 2014; 54(1): 67-79.

5. Schein EH. La cultura empresarial y el liderazgo. Barcelona: Plaza \& Janes; 1988.
6. Van Muijen JJ, Koopman P, De Witte K, et al. Organizational culture: The focus Questionnaire. European Journal of Work and Organizational Psychology. 1999; 8(4): 551-568.

7. Peiró JM. Organizaciones: Nuevas perspectivas psicosociológicas. Barcelona: PPU; 1990.

8. Gómez M. Cultura en las Pymes del sector juguete y auxiliares: Implicaciones para la implantación de sistemas de calidad. [Tesis doctoral]. Madrid: Universidad Nacional de Educación a Distancia; 2003.

9. Alonso E. El método del calibrado en la evaluación de la cultura. 8th European Congress of Work and Organizational Psychology. Verona; 1999.

10. Schneider B, Brief AP, Guzzo RA. Creating a climate and culture for sustainable organizational change. Organizational Dynamics. 1996; 24(4): 7-19.

11. Hernández R, Fernández C, Baptista P. Metodología de la investigación. 5a ed. México D.F.: McGrawHill; 2010.

12. Instituto Nacional de Estadística e Informática. Censos Nacionales 2007; XI Población y Vivienda. Lima: INEI; 2007.

13. Laguna C. Cadenas productivas, columna vertebral de los clústeres industriales mexicanos. Economía Mexicana, Nueva Época. 2010; 19(1): 119-170. 